\title{
Los registros bibliográficos y las necesidades informativas de los usuarios
}

\author{
José Antonio Frías \\ Universidad de Salamanca \\ Facultad de Traducción y Documentación
}

\subsection{Resumen.}

Se define el término registro bibliográfico en el contexto de los catálogos automatizados. Se analizan los elementos de datos contenidos en los registros bibliográficos y contemplados en las reglas de catalogación y en el formato MARC. Se constata la escasez de datos empíricos sobre la información que necesita el usuario del catálogo de la biblioteca y, tras mostrar los resultados de algunos estudios que han intentado determinar la cantidad de información que debe incluirse en un registro bibliográfico, se hace un repaso de las propuestas de catalogación de nivel mínimo y de sus efectos sobre la recuperación de la información. Se describen los objetivos, el alcance y las posibles consecuencias del Estudio sobre Requerimientos Funcionales de los Registros Bibliográficos que está desarrollando actualmente la IFLA. Finalmente, se da noticia del proyecto de registro básico puesto en marcha por el Cooperative Cataloging Council (CCC) estadounidense. (Autor)

Palabras clave: Registro bibliográfico. Elementos de datos. Reglas de catalogación. Formato MARC. Catalogación de nivel mínimo. Recuperación de la información. Necesidades de los usuarios. Estudio sobre los Requerimientos Funcionales de los Registros Bibliográficos (IFLA).

\subsection{Abstract.}

The term bibliographic record is defined whitin the context of computerbased catalogues. The data elements contained in bibliographic records, cataloguing rules, and MARC format are analized. The scarcity of empirical data on the information needed by the user of the library catalogue is shown, and after giving the results of some studies that have tried to establish the amount of information that must be included in a bibliographic record, the different proposals for a minimal-level cataloguing and their effects on information retrieval are reviewed. The objectives, scope and possible consequences of the Study on the Functional

Scire. 2 : 1 (en.-jun. 1996). 
Requirements of Bibliographic Records - which is now being developed by IFLA- are described. Then, mention is made of the core record project implemented by the American Cooperative Cataloging Council (CCC). (Author)

Keywords: Bibliographic record. Data elements. Cataloguing rules. MARC format. Minimal-level cataloguing. Information retrieval. Users' needs. Study on the Functional Requirements of Bibliographic Records (IFLA).

\section{Introducción.}

Los catálogos de la biblioteca, tal como los conocemos actualmente, están constituidos por una combinación de registros bibliográficos y de registros de fondos y localizaciones en la biblioteca, y contienen tanto menciones generales sobre las ediciones de las obras como menciones específicas sobre los ejemplares y sus localizaciones en bibliotecas concretas (Buckland, 1992, p. 24).

Al preparar un registro del catálogo, el catalogador selecciona la información significativa de la que aparece en el documento y presenta su selección de manera formalizada. En un espacio limitado y con medios tipográficos limitados debe transmitir lo esencial de lo que el impresor ha presentado, usando quizás dos o tres páginas y tipos de fuentes y tamaños distintos (Piggott, 1988, p. 72).

Para su recuperación y uso eficaces, los datos bibliográficos deben estar organizados. La primera etapa del proceso es la creación de los registros bibliográficos. El carácter esencial de un registro bibliográfico ha sido expresado por Seal, Bryant y Hall (1982, p. 8) en las siguientes palabras: "El conjunto matriz de elementos informativos de un documento bibliográfico, agrupados dentro de una estructura lógica. Cuando los datos son almacenados en forma legible por el ordenador, el registro se refiere al registro matriz del que se derivan todos los productos. Un registro se relaciona generalmente sólo con un título, del que puede haber varios ejemplares".

El registro bibliográfico ha sido definido también como "la suma de todas las áreas y elementos [...] que pueden ser usados para describir, identificar o recuperar cualquier item físico (publicación, documento) o su contenido informativo" (Anderson, 1989, p. 3).

Gredley y Hopkinson (1990, p. 9), por su parte, definen al registro bibliográfico como "una colección de elementos informativos, organizados de una manera lógica, que representan a un item bibliográfico". El item bibliográfico es definido, a su vez, como "cualquier documento, libro, publicación u otro registro de la comunicación humana; cualquier grupo de documentos o partes de un documento tratados como una entidad".

El término registro bibliográfico es relativamente nuevo, ya que ha entrado en el vocabulario de la información sobre todo como un resultado de la automatización. Mucha

Scire. 2 : 1 (en.-jun. 1996). 
de la terminología de esta área es un legado de la práctica catalográfica, reflejando la preocupación por los registros bibliográficos como registros del catálogo: asiento bibliográfico y registro bibliográfico son usados a menudo por los catalogadores con el mismo significado. Las Reglas de catalogación (1995, p. 565) no incluyen en su glosario una definición de registro bibliográfico pero definen al asiento bibliográfico como "registro o descripción, de acuerdo con unas normas, de una unidad bibliográfica o documental". El término asiento, a su vez, ha sustituido al viejo y usual de ficha, "por la variedad de soportes sobre los que actualmente se registran los datos y que ya comienzan a no ser las fichas, que se llamaban a mediados del siglo XIX, cuando fueron introducidas en el uso, papeletas o cédulas" (Carrión Gútiez, 1987, p. 132).

Sin embargo, como señalan Gredley y Hopkinson (1990, p. 10), asiento está mejor explicado en el contexto de un catálogo o bibliografía impresos, donde se refiere al listado o registro de los datos bibliográficos seleccionados relativos a un documento bajo un posible punto de acceso o encabezamiento. Los datos son presentados a partir de un registro bibliográfico y se puede hacer más de un asiento para cada documento. Esta distinción entre registro bibliográfico y asiento es más clara en los sistemas automatizados, donde el registro bibliográfico matriz se encuentra en el ordenador y los asientos son generados por uno o más programas automatizados. Los asientos pueden aparecer en bibliografías impresas o pueden ser presentados en la pantalla de los catálogos en línea.

\section{Los elementos de datos de los registros bibliográficos y las necesidades informativas de los usuarios.}

\subsection{Los elementos de datos de los registros bibliográficos.}

La información del catálogo debe ser útil para el personal bibliotecario y para los usuarios en una variedad de formas. Como señala Buckland (1988, p. 303), "la diferencia entre el catálogo bodleiano de 1620 y los catálogos contemporáneos es, esencialmente, que el último tiene una superestructura bibliográfica añadida a la información localizadora mucho mayor".

Los códigos de catalogación han dividido tradicionalmente la estructura del registro descriptivo del catálogo en dos partes: datos descriptivos y elementos que suministran acceso a esos datos (Duke, 1989, p. 121). En la actualidad, puede decirse que un registro bibliográfico se compone de:

- Descripción bibliográfica

- Puntos de acceso

- Estructura de encabezamientos

- Información de materias

- Información local (Seal; Bryant; Hall, 1982, p. 8).

Scire. 2 : 1 (en.-jun. 1996). 


\section{6}

La práctica normalizada de la catalogación consiste en presentar formalmente un número mínimo de menciones independientes que sirven para identificar y describir una publicación y para realizar una posible comparación con la descripción de otra publicación. Un lector debe, en consecuencia, poder decidir si el documento descrito en un asiento del catálogo puede responder a sus necesidades porque la descripción bibliográfica se corresponde en lo esencial a una referencia obtenida en otra parte o porque indica un documento de contenido adecuado y presentación hasta ahora desconocida para él. Los bibliotecarios además deben poder decidir si el documento catalogado era el mismo, o era significativamente distinto, que un documento del que se tiene otra descripción en el catálogo o un ejemplar concreto (Piggott, 1988, p. 72-73).

Hagler (1991, p. 26) ha señalado que los elementos de los datos bibliográficos sirven fundamentalmente para tres propósitos:

(1) para identificar un documento determinado unívocamente con el fin de distinguirlo de otros (por ejemplo, la fecha de publicación o el número de páginas de un libro impreso),

(2) para mostrar cómo uno o más documentos están asociados con otro (por ejemplo, porque tienen un autor común o porque uno es una continuación o una reimpresión de otro), y

(3) para suministrar la base para los puntos de acceso que permitirán al usuario localizar el registro de un documento en un fichero, que pueden estar relacionados con:

(a) hechos objetivos sobre el propio documento (por ejemplo, su título o el hecho de que se trata de las actas de un congreso), o

(b) lo(s) tema(s) y concepto(s) tratados en su contenido intelectual, en otras palabras, la(s) materia(s) del documento.

Gredley y Hopkinson (1990, p. 3-4) indican que teóricamente se puede establecer una división entre los datos inherentes a un documento (por ejemplo, el autor o el título) y los datos que son asignados o suministrados por diferentes organizaciones para propósitos distintos. Pero, en la práctica, esta distinción es difícil de sostener, ya que algunos datos asignados, como el título clave y el ISSN pueden actuar como identificadores únicos, estando vinculados de forma permanente a la publicación.

En ocasiones se ha establecido otra distinción de dos partes dentro del registro bibliográfico: factográfica (es decir, los datos de una citación bibliográfica tradicional) y conceptográfica (es decir, los datos que resultan del análisis del contenido del documento). Esta subdivisión procede de la división del registro bibliográfico en el campo de la descripción bibliográfica y la caracterización

Scire. 2 : 1 (en.-jun. 1996). 
bibliográfica. Sin embargo, como ha señalado Yatsko (1991, p. 48), esta división no coincide con su estructura semántica ya que "semánticamente, una descripción bibliográfica constituye una entidad coherente que incluye la anotación y el resumen y en la actualidad es la primera frase del texto de un documento secundario".

Lo que no tiene cabida en el control bibliográfico son los juicios de valor. El precio de un documento en el momento de su publicación es un elemento de la información bibliográfica en la medida en que puede ayudar a identificar un determinado aspecto o su formato, pero un juicio sobre si el precio es o no adecuado es irrelevante desde el punto de vista bibliográfico. El principal valor de un título para la mayor parte de los usuarios es que proporciona una descripción del contenido del documento y ayuda a hacer un juicio de valor sobre si le interesa o no consultarlo (Hagler, 1991, p. 27).

Las reglas de catalogación actuales permiten la representación sistemática de los datos bibliográficos de forma que éstos puedan ser recuperados y presentados de forma significativa para ayudar al usuario a encontrar y seleccionar los documentos en la colección de la biblioteca local o de otras de la red. Argumentar, como hace Lipow (1979), que las normas de entrada y las de recuperación pueden ser distintas y que las opciones de presentación a veces no están relacionadas es pasar por alto la cuestión fundamental de que las normas de entrada, de recuperación y de presentación de datos constituyen la esencia de las reglas de catalogación. En el entorno en línea, además, el catálogo de la biblioteca se ha convertido en una herramienta compleja con partes componentes interdependientes. Las normas que son formuladas para cubrir lo que pueden parecer elementos distintos e independientes en su contrapartida manual necesitan ser reevaluadas para tener en cuenta el nuevo conjunto integrado (Hancock-Beaulieu, 1991, p. 91).

Desde la introducción del formato MARC a finales de los años 60, como nos recuerda Tillett (1989, p. 149), "el diseño de los catálogos en línea ha dependido de la estructura MARC para los registros bibliográficos". Los elementos de las descripciones bibliográficas han sido cuidadosamente identificados y definidos y los formatos del registro han sido diseñados, como sabemos, con numerosos campos y subcampos. En 1968 Vickery urgía la necesidad de un estudio de las funciones de los elementos bibliográficos antes de diseñar un registro automatizado óptimo. Curran y Avram han identificado unos 400 elementos posibles. El formato MARC contempla alrededor de 250 campos y subcampos posibles (Neville, 1981, p. 140).

Más recientemente, Leazer ha presentado los resultados de un análisis de los elementos informativos de los formatos USMARC bibliográficos, de autoridades

Scire. 2 : 1 (en.-jun. 1996). 
y de fondos y localizaciones. El autor ha denunciado el alto grado de redundancia en las estructuras de los registros. Aunque no se realizó una medición estadística de la redundancia, se encontraron repeticiones obvias de elementos que aparecían en múltiples localizaciones en los formatos. Informaciones como el lugar y la fecha de publicación son registradas en diferentes campos (24 y 58 campos diferentes en los tres formatos respectivamente). Se encontraron repeticiones similares de otros tipos de datos distintos en los tres formatos, por ejemplo, la localización del documento catalogado (ocurre en 11 campos independientes), la información de la edición (18 campos) y la forma de la composición musical (26 campos, sin incluir los que expresan contenido temático).

Incluso la información específica es registrada en múltiples lugares. La frecuencia de publicación fue localizada en 8 campos independientes, la información sobre procesamiento y preservación en 6 campos, la información sobre préstamo y acceso en 9 campos e incluso la expresión de la presencia de datos matemáticos es localizada en 2 campos. Alguna información específica, como las características del fichero para los archivos de ordenador, es localizada solamente en un campo específico creado para este dato, sin incluir los campos de notas globales (Leazer, 1992, p. 196).

\subsection{Las necesidades informativas de los usuarios.}

El reciente desarrollo de las redes bibliográficas, el coste en aumento de productos como ficheros que incrementan su tamaño, el uso de registros MARC menos completos en programas CIP, el rápido crecimiento de las bases de datos para la recuperación de la información en línea y otros desarrollos han hecho más acuciante la necesidad de más información sobre los méritos relativos de los catálogos de asientos completos y abreviados. La investigación previa ha estado limitada porque raramente ha usado técnicas experimentales y no ha tenido en cuenta los distintos factores implicados en su interacción (Seal, 1983, p. 145).

La importancia del conocimiento de las necesidades informativas de los usuarios ha sido puesta de manifiesto por Lehmann (1992, p. 88), para quien "es necesario un mejor conocimiento de las necesidades de los usuarios, además de una diferenciación de los requerimientos mínimos y los requerimientos deseables [...] Debemos fijar diferentes niveles bibliográficos, para permitir que sean usadas definitivamente por ciertos materiales (por ejemplo, normas completas y mínimas)”.

La cantidad de información que debe incluirse en un registro bibliográfico o un asiento del catálogo ha sido un tema de debate tradicional en el mundo bibliotecario. Un alto nivel de contenido de datos como el del formato MARC se logra, en opinión de Butcher (1988), no sólo con el coste de la rapidez sino además con otros costes que se están incrementando de forma inaceptable para el mundo del

Scire. $2: 1$ (en.-jun. 1996). 
libro. Como señala el autor, "la lista de desventajas parece crecer en proporción directa al crecimiento del nivel de contenido de datos en los propios registros" (ibidem., p. 108).

Bryant (1988, p. 15), al hacer un análisis de los requerimientos de los usuarios finales respecto a los registros bibliográficos, ha señalado que el contenido de un registro del catálogo afecta al sistema bibliotecario y a los usuarios de la biblioteca en tres áreas principales: costes del sistema, necesidades del usuario y utilidad.

(1) Costes del sistema: puede argumentarse que los catálogos con asientos más breves significan un ahorro en los costos.

(2) Necesidades del usuario: contrapesando esta consideración tiene que decidirse si los datos que se dejan fuera de los registros son realmente necesarios para los usuarios (lectores o personal bibliotecario).

(3) Utilidad: está relacionada con la facilidad con la que se puede realizar la búsqueda en el catálogo, especialmente con la rapidez y la precisión de la búsqueda y la preferencia del usuario.

Contrariamente a lo que parecen creer algunos catalogadores y bibliógrafos, Line (1988, p. 10) señala que el registro bibliográfico no es el summum del logro humano, incluso superior al creativo trabajo artístico al que se refiere. Es "un medio para un fin, y sólo un medio para un fin". En su opinión, muchos registros del catálogo de la biblioteca, y muchos códigos de catalogación, han sido diseñados sobre todo para satisfacer la "neurosis perfeccionista de los bibliotecarios". Estos códigos, al menos hasta hace poco tiempo, han sido elaborados sin ningún intento serio de averiguar lo que quieren los usuarios, sino sólo lo que necesitan (1) e, incluso, sin ningún intento de probarlos respecto a su aceptación y utilidad para los usuarios (2). Tyckoson (1991, p. 37) ha señalado que mientras "los usuarios tienden a considerar la biblioteca como suministradora de informaciones, nosotros tendemos a considerarnos recopiladores de informaciones".

Tradicionalmente, los bibliotecarios han tendido a pensar que la principal función del catálogo debía ser la de permitir al usuario determinar si la biblioteca tenía un documento determinado, qué obras de un determinado autor existen en la colección, qué ediciones de una obra determinada tiene la biblioteca y qué materiales posee ésta sobre una materia concreta. En definitiva, un registro debe dirigir al usuario hacia un documento determinado en la colección, mostrar al usuario su localización, su descripción física y sus contenidos temáticos. Esto muestra, como ha señalado Hufford (1991, p. 36), que las necesidades del usuario y su conducta "vis-à-vis" con los catálogos de la biblioteca pueden ser valiosas para que se cumplan estos propósitos. Y, si esto es así, el uso del catálogo debería jugar un papel en el desarrollo de la teoría y la práctica de la catalogación.

Scire. 2 : 1 (en.-jun. 1996). 
El conocimiento del uso puede aportar una base empírica importante para la catalogación. Aunque los autores de los códigos pretéritos tuvieron presente al usuario cuando los elaboraron, no hay evidencia de ningún estudio empírico del uso de las informaciones específicas de los registros bibliográficos que haya afectado a alguno de estos códigos (3).

El catálogo en línea ha fomentado y facilitado el estudio de los hábitos de búsqueda y las necesidades de los usuarios. Los OPACs nos proporcionan "una información muy valiosa para poder solucionar tanto los problemas de comunicación del sistema con los usuarios, como los problemas relacionados con el contenido de los registros y las formas de acceso a los mismos" (García Caro, 1994, p. 420-421). Sin embargo, algunos problemas de la práctica diaria de los catalogadores siguen estando basados en "distinciones que parecen importantes para los catalogadores pero que no ayudan a los usuarios a encontrar la información que buscan" (Hyslop, 1994, p. 35). Magán Walls (1995, p. 192), en la misma línea, insiste en que:

[...] la catalogación [...] sigue siendo uno de los procesos que menos han evolucionado en los últimos años para adaptarse a las necesidades de los usuarios. Así, los últimos trabajos sobre OPACs inciden precisamente en la presencia de información bibliográfica de gran importancia para los profesionales y bibliógrafos pero de escasa utilidad para el usuario medio.

Debe existir una relación entre el análisis del uso del catálogo y los datos catalográficos. Por ejemplo, aunque la frecuencia de consulta de un determinado elemento bibliográfico puede no ser razón suficiente para ser incluido o excluido de un código y de los registros creados utilizando ese código, sí es un medio útil para relacionar el uso de los catálogos con la creación de nuevos códigos. Esta idea no es nueva. En 1968 Michael Gorman (p. 66-67) hacía constar que:

[...] el aspecto más vital de la teoría y la práctica de la catalogación que permanece sin ser examinado es el uso que se hace del catálogo. Hasta que la finalidad de la construcción del catálogo sea claramente definida sobre la base de encuestas objetivas y precisas del uso del catálogo, toda la teoría de la catalogación seguirá siendo acientífica y abierta a la duda.

Pese a la publicación de las AACR2 en 1978 y su edición revisada (AACR2R) en 1988, la cuestión de si debe darse tal relación ha seguido ignorándose (Hufford, 1991, p. 36). Boll (1990, p. 3) ha sugerido que en estos momentos es necesario "un examen completo de los objetivos y principios de todos los aspectos de la catalogación en la era de la informática". Una de sus pre-condiciones para un código nuevo es que "debe tener en cuenta los hallazgos de investigaciones recientes sobre el uso del catálogo [y] estudios generales de usuarios" (ibidem). El reconocimiento de que existe una importante relación entre los códigos de catalogación y estos estudios ha sido largamente ignorado en la profesión.

Scire. 2 : 1 (en.-jun. 1996). 
Los datos acumulados derivados de las encuestas de uso del catálogo que emplean técnicas de investigación válidas y apropiadas deben ser consultados siempre que los bibliotecarios profesionales consideren la revisión de códigos de catalogación, ordenación de catálogos públicos y/o el contenido de los registros bibliográficos (Hufford, 1991, p. 36).

En este sentido, muchos de los estudios de los OPACs se han concentrado en la eficacia del proceso de búsqueda de los usuarios. La representación de los juicios de éstos sobre la utilidad de los documentos es fundamental para el desarrollo de métodos y técnicas seguros para medir la eficacia de los sistemas de recuperación de la información. Los resultados de un buen número de estos estudios han mostrado que, aunque los usuarios están entusiasmados, los sistemas no son explotados totalmente y las citas de documentos relevantes no son siempre fáciles de recuperar.

Generalmente, los juicios de los usuarios son descritos usando el concepto de "relevancia". Pero dichos estudios, como señala O’Brien (1990, p. 269), "se han centrado en una mezcla a menudo indiferenciada de relevancia objetiva y subjetiva". Un documento es relevante si el usuario considera que el documento es útil, de lo contrario no sería relevante. Basadas en tales decisiones dicotómicas, han sido propuestos una serie de criterios de eficacia como la precisión, la exhaustividad, el índice de irrelevancia, la precisión y la exhaustividad normalizadas y la esperada búsqueda extendida. Sin embargo, el uso de la noción dicotómica de "relevancia" ha provocado cierto criticismo hacia estas medidas (Yao, 1995, p. 133).

En opinión de Park (1993, p. 319), pese a la riqueza de la literatura sobre la "relevancia", no existe una definición comúnmente aceptada. Aunque mucha de la investigación experimental en recuperación de la información está basada en la idea de la relevancia, se han hecho distintas interpretaciones del concepto. Debido a su papel clave en la investigación, la falta de consenso sobre el significado de la relevancia y la "evaluación de la relevancia" plantea cuestiones de carácter metodológico.

Haciendo una distinción entre relevancia objetiva y subjetiva, Swanson (1986) definió la "relevancia subjetiva" como la experiencia mental de una persona concreta que tiene una necesidad informativa. La "relevancia objetiva" fue definida como una relación lógica entre un documento y una mención escrita objetivizada de una solicitud. La relevancia, en este sentido, es una conexión entre una solicitud escrita y un documento y pertenece al mundo del conocimiento objetivo.

Además, como señala Stubley (1988, p. 46), decir que son los usuarios de la biblioteca quienes dictan los requerimientos a los registros MARC es una cosa

Scire. 2 : 1 (en.-jun. 1996). 
pero definir estos requerimientos es, a menudo, algo muy diferente, ya que los usuarios pueden aportar una serie de factores a la búsqueda en el catálogo: su propia terminología, experiencia, formación y, sobre todo, las necesidades específicas del trabajo concreto. Parece algo comprobado que el personal de los servicios de lectura tiene un conocimiento de los principios bibliotecarios generales considerablemente superior al de los usuarios, pero su conocimiento y experiencia de técnicas como la búsqueda en línea no es necesariamente superior.

Bruce (1994) ha presentado una investigación basada en la asunción de que los usuarios determinan la relevancia de los documentos recuperados en un sistema de información de acuerdo a una síntesis intelectual de respuestas a una combinación de estímulos operativos, como el documento y los atributos de la información de los registros del documento. Las características del documento son: autor(es), título, descriptores de materia, fuente de publicación y fecha de publicación. Los atributos de la información son: la exactitud, lo completo, el contenido, la capacidad de sugerencia, la rapidez y el tratamiento (ibidem, p. 144).

Abrera (1982) realizó un experimento práctico para determinar los requerimientos del control bibliográfico de una biblioteca pública de tamaño mediano o pequeño desde el punto de vista de las necesidades expresadas por los usuarios a través del teléfono y del servicio de referencia. Se midieron todos los elementos así como la extensión y el uso de cada uno de ellos para la recuperación. Los resultados del estudio de los requerimientos de los usuarios y la naturaleza de su estructura bibliográfica indicaron que los datos bibliográficos presentes en un registro catalográfico suministraban información suficiente para satisfacer las necesidades de los usuarios y que no existía una necesidad inmediata para aumentar el acceso más allá de los niveles ofrecidos en ese momento por el catálogo de la biblioteca.

Aunque se trataba de un catálogo de fichas, un estudio realizado por Osiobe (1987) entre los estudiantes de la University of Port Harcourt puso de manifiesto que la unidad de información considerada menos útil en el catálogo era el área de descripción física del material $(43,7 \%)$. Considerando la preponderancia de la búsqueda de publicaciones conocidas sobre otros tipos de búsquedas, Osiobe no considera sorprendente el bajo porcentaje de utilidad de la información de la descripción física en la ficha del catálogo puesto que muchos usuarios estaban ya familiarizados con el formato del documento que buscaban. Una explicación alternativa puede ser que los usuarios de la biblioteca simplemente no tienen interés en esa información.

Un aspecto sorprendente del estudio es el alto porcentaje otorgado a la información de las notas suplementarias en términos de utilidad: 287 usuarios $(57,5 \%)$ consideraron útil la información del área de notas. A juzgar por el porcentaje de

Scire. $2: 1$ (en.-jun. 1996). 
respuestas, puede observarse que la información ofrecida en las notas de las fichas del catálogo por los bibliotecarios ha sido la que más ha ayudado a los usuarios.

Un porcentaje mayor que el área de notas en términos de utilidad tiene el área de edición: 293 usuarios $(58,7 \%)$ consideraron útil la información de la edición. La información de la serie fue considerada más útil $(55,3 \%)$ que la información sobre la publicación $(54,1 \%)$.

De todas las unidades de información establecidas en la ficha del catálogo sólo fue considerada inútil por más de la mitad de los estudiantes la información sobre la descripción física. Las demás unidades de información fueron consideradas útiles por más de la mitad de los usuarios.

\section{La catalogación de nivel mínimo y la eficacia de la recuperación.}

Una descripción realizada con un nivel de detalle completo contiene suficiente información para identificar un documento y para "dar alguna idea de su adecuación para un uso concreto" (Piggott, 1990, p. 12), al margen de cualquier encabezamiento que pueda asignársele para el propósito de determinar su lugar y facilitar su encuentro en el catálogo. No obstante, para algunos catálogos y para algunas formas de utilización del catálogo, como hemos comentado, parece adecuada una cantidad más pequeña de información. Cuando el objetivo principal del usuario que consulta el catálogo es la localización de una publicación conocida, a menudo es suficiente una forma abreviada del asiento, y en un catálogo de autores, cuando el encabezamiento asignado es el nombre de un autor, que se repite en la mención de responsabilidad el documento catalogado, puede no ser necesario repetir el mismo nombre en la descripción.

Se han desarrollado varios intentos de identificación de los elementos esenciales y no esenciales de los asientos del catálogo (4). Las primeras investigaciones se basaban en la interpretación de las opiniones de lo usuarios del catálogo sobre sus necesidades, desde la comparación directa, durante el uso normal, de catálogos con registros de diferentes niveles de extensión, ya que se instalaban, con propósitos experimentales, catálogos paralelos conteniendo subconjuntos diferentes de los mismos registros MARC. El Bath Centre for Catalogue Research (desde noviembre de 1987 el Centre for Bibliographic Management) realizó experimentos en diferentes bibliotecas, con la esperanza de obtener resultados comparativos que pudiesen ser válidos estadísticamente. Los objetivos primordiales de los investigadores fueron: (1) ver en qué medida los asientos de monografías del catálogo, construidos con diferentes niveles de contenido, permitían satisfacer las necesidades de los usuarios y se podían usar con facilidad; (2) encontrar si existía un subconjunto en lengua inglesa del formato MARC

Scire. $2: 1$ (en.-jun. 1996). 
generalmente aceptable; y (3) establecer una posible relación de tal subconjunto, si se encontraba, con el programa CIP (Seal; Bryant; Hall, 1982, p. 8).

Los resultados del Centre sugerían que el $97 \%$ de las necesidades de los usuarios, tanto bibliotecarios como lectores, podían ser satisfechas con número muy reducido de elementos de la descripción, en contraste con el asiento completo compilado en muchas bibliotecas. El informe del Centre recomendó la retención del encabezamiento de nombre, ya sea éste personal, corporativo o un título uniforme; el título y el subtítulo, el volumen o el número de parte; con el volumen o título de parte; mención de edición; fecha de publicación; una nota de la relación de la publicación con otra obra y de su historia bibliográfica, y el ISBN u otro número de control. Se omiten completamente la colación y la mención de serie. Debía hacerse un asiento de título, y no deberían restringirse los asientos secundarios bajo nombres personales o corporativos ni sus referencias. Debían ofrecerse encabezamientos de materia personales y corporativos en el catálogo de nombres. El informe estimaba que un catálogo de este tipo permitía ahorrar un $40 \%$ de los costos de producción de un catálogo COM de nivel completo. Los elementos del asiento eran casi idénticos a los del asiento CIP, ya que ambos fueron comparados. El informe encontró que un asiento de este tipo podía ser satisfactorio para muchos usuarios, aunque los materiales y las circunstancias en otras bibliotecas podían requerir una catalogación más detallada.

Bryant (1995, p. 31) recordaba recientemente que la calidad es asimilada a menudo a la exhaustividad de la descripción bibliográfica, ahora que el desarrollo de los OPACs y de su acceso en redes nacionales e internacionales ha conducido a una demanda de más información sobre el contenido de los libros y los documentos. Sean cuales fueren las concepciones sobre el nivel de detalle en un registro bibliográfico, el autor piensa que "muchos estarán de acuerdo sobre los parámetros esenciales de la calidad de los asientos: precisión, coherencia, puntualidad, audiencia y funcionalidad, es decir, la adecuación de los registros a las necesidades que deben satisfacer al usuario".

Reconociendo que los ahorros en catalogación se conseguirán a través de la selección de entre todos los datos recomendados (de acuerdo a las ISBD, AACR u otros códigos de catalogación) para la catalogación detallada de grandes colecciones (y que ha sido la catalogación realizada durante más de un siglo), las propias AACR2 (y nuestras Reglas de catalogación) buscan asegurar un contenido mínimo esencial y una secuencia normalizada en los asientos del catálogo hechos por bibliotecas individuales y mantener la compatibilidad entre bibliotecas que tengan diferentes requerimientos al prescribir tres niveles de detalle admisibles en la descripción.

El nivel más bajo es significativamente más completo que el asiento catalo-

Scire. 2 : 1 (en.-jun. 1996). 
gráfico "breve" para monografías que el Bath Centre for Catalogue Research consideraba que satisfacía al 97\% de los usuarios (Piggott, 1990, p. 14). Incluye adicionalmente otros elementos como los datos específicos de la clase de documento (en el material que corresponda), el primer editor, la descripción física básica y las "notas" están indefinidas. Estos términos son necesariamente generales, puesto que se refieren a todos los tipos de materiales bibliotecarios.

El segundo nivel de descripción de los códigos actuales incrementa muchos de los campos en el asiento (el título y la mención de responsabilidad, la publicación, la descripción física) y añade la mención de serie. El tercer nivel de la descripción incluye todos los elementos previstos en los códigos que sean aplicables al documento de que se trate.

La elección de uno u otro nivel lo realizará cada centro, a la vista de sus necesidades y de la importancia de sus colecciones, "bien adoptando el mismo nivel para todos sus fondos, bien utilizando distintos niveles de detalle según el tipo de documento o, incluso, aplicando un criterio selectivo dentro de los de un mismo tipo, de acuerdo con determinadas características que en ellos puedan darse" (Reglas de catalogación, 1995, 1.0.9).

En 1982, en un esfuerzo para mejorar la tasa de éxito, la British Library desarrolló mucho su programa CIP, pero esto no produjo resultados apreciables. Durante varios años, las tasas de éxito permanecieron aproximadamente entre 62-64\% y, a mediados de los 80, la situación de los atrasos en catalogación alcanzó proporciones críticas. En 1987 la BL publicó un documento consultivo titulado Currency and coverage que, entre otras iniciativas, proponía un nivel más bajo de descripción bibliográfica para ciertas categorías de documentos con el fin de permitir el reparto de los recursos para acelerar la producción de registros. Esta política fue adoptada para los registros BNBMARC en enero de 1988 y permitió mejorar de manera sensible la "puntualidad", reduciendo el detalle en la catalogación en la mitad de los asientos. Ciertos tipos de materiales son catalogados ahora siguiendo las AACR2 para la descripción de nivel 1, con la adición del precio y la información sobre la accesibilidad. Los materiales catalogados de esta manera son los libros ingleses de ficción modernos, los libros infantiles, los folletos o publicaciones con menos de 33 páginas y obras de ciencia, tecnología y religión. El resto de los asientos continúan siendo catalogados de acuerdo al nivel 2 de las AACR2 con la adición del precio y la información sobre la accesibilidad (Piggott, 1990, p. 14-15). Esta decisión suscitó, no obstante, numerosas críticas procedentes no solamente de los bibliotecarios, sino también de la industria editorial y de comercio del libro (Bryant, 1995).

Harris (1989) ha estudiado las tendencias evolutivas en el tamaño de los registros. Para determinar si existía una tendencia antes de 1982 hacia los regis-

Scire. 2 : 1 (en.-jun. 1996). 
tros catalográficos más breves, comparó 99 muestras del National Union Catalog Pre-1956 Imprints y el National Union Catalog (1982), encontrando que el número de caracteres se incrementó en un $24,5 \%$, el número de puntos de acceso en un $130,2 \%$, el número de campos en un $96,9 \%$ y el número de subdivisiones de los encabezamientos de materia en un $156,2 \%$. Los datos se recogieron antes del aumento de catalogación de nivel mínimo que se produjo a partir de mediados de los 80 . Además, el autor ha incluido un análisis de costes en su estudio.

Con el incremento de los atrasos en algunas grandes bibliotecas, junto con el alto coste de la catalogación de un documento, la catalogación de nivel mínimo se ha convertido en "un refugio o una salvación para los administradores de los departamentos de catalogación y servicios técnicos" (Esman, 1991, p. 3). Algunas grandes bibliotecas de investigación anglosajonas han usado la catalogación de nivel mínimo como una herramienta prioritaria para reducir y eliminar los atrasos. Igualmente, algunas bibliotecas pequeñas que carecen tanto de experiencia en catalogación como de personal para completar la catalogación crean registros breves para algunos años. Esta tarea puede llevarse a cabo en una época en que la tecnología está ampliando las posibles funciones de la biblioteca. La exigencia de una simplificación de la catalogación es muy relevante pero debe tener en cuenta todas las funciones presentes y futuras que deseamos que realice el catálogo de la biblioteca.

La catalogación de nivel mínimo ha sido propuesta como la forma más directa de disminuir los costes mediante la eliminación de algunas partes del registro del catálogo. Aparte de la eliminación de los encabezamientos de materia, puede acortarse la propia descripción. Un peligro es que, como señala Knutson (1993, p. 262), "si no existe un acuerdo general bien fundado sobre qué elementos ISBD deben constituir la descripción mínima, algunas bibliotecas nacionales pueden iniciar diferentes políticas".

En respuesta a esta situación, Lambrecht (1992) ha llevado a cabo un estudio sobre la catalogación de nivel mínimo realizado por las agencias bibliográficas nacionales. Sorprendentemente ha dado cuenta del escaso consenso existente sobre qué debe constituir una descripción mínima, pues sólo se pusieron de acuerdo en considerar como obligatorios 4 de los 20 elementos ISBD obligatorios en la actualidad (título propio, mención de edición, nombre del editor y fecha). Otros 9 elementos, como la información complementaria sobre el título y la mención de responsabilidad, son considerados necesarios o deseables. Lambrecht recomienda convertir en opcionales otros 7 elementos, incluyendo la mención de ilustraciones y el ISSN de la serie.

En otra parte del libro, igualmente importante, Lambrecht hace hincapié en algunos defectos de los argumentos avanzados sobre la catalogación de nivel

Scire. 2 : 1 (en.-jun. 1996). 
mínimo. La investigación sobre la materia realizada desde los estudios de la University of Bath a principios de los años 80 es muy poco sólida: no sabemos mucho del efecto en el acceso del usuario o del volumen de ahorro que supone. La supresión de elementos descriptivos puede tener sólo un efecto marginal sobre los costos de la catalogación y, al mismo tiempo, limitar lo que una biblioteca puede desear hacer en un futuro para mejorar su catálogo. En este sentido, Beheshti (1992) ha demostrado cómo incluso la descripción física de un libro (uno de los objetivos preferidos de la simplificación) puede tener un nuevo uso si se incorpora en la pantalla de un catálogo en línea orientado a objeto.

Una perspectiva más completa puede obtenerse a través de la práctica. Fox y Preece (1991) han estudiado las implicaciones de la mejora de la catalogación de nivel mínimo por medio del análisis de 190 de los 200 registros de nivel mínimo introducidos en un departamento de catalogación durante el período de un año. Éstos fueron buscados en OCLC para los cambios cuatro meses después del comienzo del proyecto. En ese momento, el $25 \%$ habían sido cambiados o reemplazados por registros de la LC. Al final del proyecto, la biblioteca había mejorado 134 registros $(70 \%)$, mientras otras bibliotecas habían mejorado 56 registros (30\%). Se determinó el tiempo que cada registro estuvo en la base de datos (ILLINET Online) antes de ser mejorado. La escala varió entre una semana y 124 meses, con una media de 24 meses y una mediana de 9 meses. El número de cambios realizados en los campos variables alcanzó una media de 8,24 por registro para el conjunto completo (los campos MARC 6xx fueron los más modificados o añadidos). Los datos indicaron que la mejora requiere la revisión del registro completo, que las pautas para la catalogación de nivel mínimo son aplicadas de forma inconsistente por otras bibliotecas y que la mejora requiere un personal de alto nivel, con "un conocimiento sustancial de los códigos de catalogación y de materias" (ibidem, p. 31).

Patton (1991), por su parte, ha realizado una completa y documentada historia del desarrollo de la catalogación menos completa y los registros de nivel K (nivel mínimo) en OCLC. En el momento de redactar su estudio (abril de 1990), los registros de nivel $\mathrm{K}$ de todos los formatos representaban el 18\% de la base de datos de OCLC. Si se comparan estos datos con el $11 \%$ aproximado de mediados de 1985 y el 2,6\% de mediados de 1978 se observa un incremento sostenido que refleja las presiones sobre las bibliotecas para que ofrezcan algún nivel de acceso a los materiales no catalogados. En el mismo período de tiempo, sin embargo, el porcentaje de registros de materiales audiovisuales de nivel $\mathrm{K}$ disminuyó del 57,4\% en 1978 al 19,5\% en 1985 y al 19,4\% en 1990 (ibidem, p. 29). Dependiendo, por tanto, del punto de vista que se adopte, estos datos pueden ser interpretados como "más" y "menos".

Handman y Horny han llevado a cabo dos estudios distintos sobre la catalo-

Scire. 2 : 1 (en.-jun. 1996). 


\section{8}

gación de nivel mínimo de materiales audiovisuales. Ambos autores recomiendan cautela al tratarse de un medio en el que la búsqueda de documentos conocidos es rara. No debe olvidarse que no se produce ningún ahorro si los usuarios no pueden localizar los materiales seleccionados. Handman (1991) ha señalado la tendencia dentro de la comunidad de catalogadores a tratar estos materiales de forma diferente a las publicaciones impresas, proporcionando una catalogación menos completa. El autor discute el impacto de la catalogación de nivel mínimo en la capacidad del usuario para recuperar estos materiales, citando ejemplos donde el acceso a éstos es impedido en gran medida por la ausencia de asientos secundarios, encabezamientos de materia y encabezamientos de género.

En 1986, la biblioteca de la Northwestern University comenzó a adquirir videos que podían servir de apoyo a los programas docentes de la universidad. Con la expectativa de recibir 600 nuevos videos, el ahorro de tiempo en la catalogación se convirtió en una cuestión primordial. En vista del deseo de continuar reduciendo los atrasos existentes en la catalogación, un grupo de trabajo sobre catalogación de videos llevó a cabo un estudio minucioso para determinar qué datos del registro bibliográfico eran necesarios y cuáles no. Después de varias consultas, se acordó un registro catalográfico de nivel completo modificado. Estos registros, descritos por Horny (1991), son diseñados para atender los requerimientos del personal y los usuarios teniendo en cuenta las ventajas de las capacidades de la búsqueda en línea, con el resultado de que están lejos del registro reducido asociado normalmente con la catalogación de nivel mínimo.

Soules, Lucas y Pritts (1992) han informado sobre un proyecto de la University of Michigan para simplificar la catalogación de los documentos de trabajo usando RLIN de forma que los estudiantes en prácticas puedan hacer el trabajo utilizando una "plantilla". Aunque el registro es breve, respeta las AACR 2 con dos excepciones. La primera es que todos los asientos son tratados como asientos principales de título para eliminar la necesidad de que los estudiantes tengan que elegir el punto de acceso principal. Esta decisión no se consideró un problema porque la automatización es inminente y un catálogo en línea reduce la naturaleza crítica del asiento principal. Todos los autores se recogen en el registro, permitiendo al distribuidor que realiza la lista mensual de adquisiciones recientes de la biblioteca la impresión de los nombres de los autores como parte del asiento.

Oddy, en una conferencia reciente, se apartó del consenso cooperativo y de los planteamientos basados en el mercado que han sustituido la construcción a largo plazo por la satisfacción de las necesidades a corto plazo (Trickey, 1995, p. 6). En la misma línea, Gorman recuerda que, cuando se habla sobre simplificación, se olvida que existen tres niveles de descripción, uno de los cuales es bastante exiguo, "es una descripción muy corta y ofrece a las personas puntos de

Scire. $2: 1$ (en.-jun. 1996). 
acceso por el nombre por el que seguramente realizarán la búsqueda y suministra referencias desde los otros nombres" (Question and answers, 1994, p. 44). Brunt (1992, p. 26), al estudiar la adecuación de los códigos de catalogación a la recuperación de la información en los OPAC va aún más lejos y afirma que el programa ISBD "necesita ir hacia la inclusión, más que hacia la exclusión, de elementos informativos".

En este sentido, Bourdon (1993, p. 87-88), en su estudio sobre los registros de autoridad producidos por las principales agencias bibliográficas nacionales, ha señalado que el contenido de los registros bibliográficos es tan importante para efectuar el control de los puntos de acceso de autor como el contenido de los registros de autoridad. Son sus correlaciones las que permiten atribuir correctamente las obras a los autores que son responsables de éstas. La autora señala que no es inútil volver a llamar la atención sobre esta evidencia en un momento que se apresta a abreviar las descripciones bibliográficas. En su opinión, suprimir la mención de responsabilidad del registro bibliográfico, bajo el pretexto de que el nombre del autor figura en el encabezamiento, es privarse de un elemento de identificación a la vez del documento y del autor, al menos cada vez que la forma del nombre que aparece en la obra sea diferente de la forma retenida en el encabezamiento. Incluso suprimir las menciones de traducción o de título original es hacer más ardua la tarea de los catalogadores que reutilizan estos registros bibliográficos incompletos en otro catálogo donde la lengua del autor podría ser un elemento de identificación útil en caso de homonimia. Estas abreviaciones de las ISBD deben, pues, ser abordadas con prudencia, sobre todo si se tiene la intención de aplicarlas en las bibliografías nacionales. Respecto a estas últimas, Karamac (1992, p. 75) ha señalado que "han sido y deben seguir siento un núcleo en la cadena de la información". En su opinión, un nivel alto de normalización de todos los componentes o vínculos de la cadena parece ser un requisito previo para nuestro éxito resolviendo las necesidades de los usuarios.

Una buena síntesis del estado de la cuestión ha sido realizada por Busquets (1992) y puede ser expresada en el siguiente párrafo:

La aceptación y el consenso definitivo en torno a las nuevas opciones y/o estándares no va a ser fácil. Existe diversidad de opiniones al respecto. Hay quienes opinan que debe mantenerse el nivel actual: se trataría de obtener los suficientes recursos económicos. Por otra parte, están quienes creen que el entorno automatizado y la evolución acelerada de las tecnologías de la información determinan factores técnicos y metodológicos para una reevaluación y/o adaptación de estándares, que se suman a los económicos. El último criterio parece, de momento, ser el que predomina entre influyentes sectores de la comunidad profesional y servicios bibliotecarios concretos (ibidem., p. 170).

Podría añadirse finalmente que el estado actual de los catálogos en línea, sin

Scire. 2 : 1 (en.-jun. 1996). 
embargo, con bastantes diferencias entre ellos -sobre todo como mecanismos para recuperar registros- es "una barrera real para tales cambios" (Byrum, 1992, p. 139).

\section{La IFLA y los requerimientos bibliográficos funcionales.}

La inquietud que se percibe en el mundo bibliotecario no es privativa de éste. Durante los últimos años lo que podríamos denominar "mundo del libro" (editores, agencias bibliográficas, libreros y proveedores de bibliotecas, bibliotecarios) han buscado una estrategia conjunta de autodefensa. Como ha señalado Graham (1988, p. 16), "la era de la información electrónica nos está forzando a abandonar el sectarismo del mundo impreso". Se ha producido un movimiento amplio entre los bibliotecarios para usar el procesamiento de datos electrónicos y la comunicación para reducir la duplicación del trabajo e incrementar el uso de registros creados cooperativamente, bien dentro de una organización particular, donde puedan integrarse la selección, adquisición, catalogación y circulación de registros o bien entre bibliotecas a través de esquemas de red.

En muchas de las discusiones y debates suscitados en los múltiples encuentros y seminarios celebrados se planteó la posibilidad de un consenso sobre el contenido bibliográfico de un registro que pueda ser generado por un editor y usado (con adiciones o sustracciones) por los libreros, bibliotecarios y usuarios de la biblioteca para sus propios propósitos (Piggott, 1990, p. 290). Un resumen de las discusiones de la década de los 80 realizado por Dempsey (1989) incluye una tabla identificadora de los elementos de un registro de este tipo que pueden ser necesarios para cada grupo. Dempsey identifica un conjunto básico de elementos de datos: ISBN, título, autor, edición, editor/impresor, fecha de publicación, encuadernación y precio. Indica los elementos requeridos en tres categorías: (1) descripción y control bibliográfico, es decir, la identificación y la descripción del ejemplar y un encabezamiento normalizado siempre que éste se encuentre; (2) descripción del contenido para la selección y promoción, incluyendo la afiliación del autor, el nivel de lectura, la lista de contenidos, el sumario y las palabras clave; y (3) información de materias, usando la clasificación, los encabezamientos de materia (más valiosos si pueden asignarse a partir de un tesauro acordado), y palabras-índice, algo que se está convirtiendo en algo necesario en las bases de datos bibliográficas, frecuentemente accesibles en CDROM, que son consultadas con fines de referencia. Dempsey añade que "el aparato (tecnológico, comercial y político) no existe ya para la difusión de datos dentro de un sistema continuo [...] Las normas para la descripción y el intercambio electrónico de datos bibliográficos a través del mundo del libro en su conjunto sólo se han convertido en una materia de interés recientemente".

En 1988, el International MARC Network Committee of Directors of

Scire. $2: 1$ (en.-jun. 1996). 
National Libraries realizó una encuesta bibliográfica a sus miembros con resultados preocupantes. No se consiguió una cobertura amplia y únicamente fue satisfactoria para los materiales impresos, en concreto para las publicaciones comerciales. Pareció existir una tendencia a la reducción en las normas de catalogación. Esto, como señala Clement (1992, p. 2), "puede ser un aspecto sensible y efectivo económicamente pero existe el peligro de que las normas puedan reducirse por debajo del nivel que permite el uso eficaz de los registros por otros". Los creadores de registros nacionales habían tomado algunas decisiones muy duras, a menudo incluyendo las concesiones mutuas entre los distintos objetivos del CBU. Otra conclusión que podía desprenderse de la encuesta era que las bibliotecas nacionales no podían completar solas todos los objetivos de la creación de registros bibliográficos completos, actualizados, baratos y de calidad.

En este contexto, el Programa UBCIM de la IFLA patrocinó un Seminario sobre Registros Bibliográficos, celebrado en Estocolmo en 1990 con un objetivo básico: "examinar el propósito y la naturaleza de los registros bibliográficos y el rango de necesidades que puede esperarse que sean cubiertas de forma realista para encontrar y considerar formas alternativas de cubrir estas necesidades de una forma barata y cooperativa" (ibidem).

La discusión en el Seminario de Estocolmo se centró en la calidad y las funciones de los registros bibliográficos. Algunos intervinientes propusieron que la catalogación se simplificase considerablemente. Uno de los participantes indicó que el número de elementos de datos descriptivos podía reducirse sin afectar seriamente al acceso. Esta mención se basaba en los estudios de uso del catálogo de la University of Bath. Además, se mencionó que las ventajas económicas podían producirse distribuyendo la tarea de la construcción de los registros bibliográficos, en concreto se sugirió que los consumidores de registros bibliográficos, como libreros y editores, podían participar en el suministro de elementos para crear registros genéricos. Otro participante, en sentido contrario, señaló que las propuestas anteriores no tenían en cuenta la variedad de las funciones que un registro bibliográfico debe realizar. Además, el coste del suministro de control bibliográfico no reside tanto en la provisión de elementos de datos descriptivos como en la provisión de la organización de los elementos de datos que requieren control de autoridad (Tillett, 1995).

Al final del seminario no existía consenso sobre las funciones de los registros bibliográficos y se hizo evidente para los participantes la necesidad de un reexamen fundamental del registro bibliográfico. Como señaló Chevallier (1992, p. 46), "son urgentes y necesarias informaciones complementarias sobre las prácticas nacionales y la promoción de un estudio por parte la IFLA para dar una oportunidad a los distintos debates que no han tenido lugar, con el resultado final de la publicación de unas nuevas pautas de catalogación que sean adoptadas por

Scire. 2 : 1 (en.-jun. 1996). 
todos los países". En concreto, se consideró necesario un acuerdo común sobre los objetivos del registro bibliográfico como proveedor de información y sobre cómo consigue responder a las necesidades de los usuarios. Para iniciar este reexamen se propuso el estudio de los requerimientos funcionales de los registros bibliográficos.

Esta cuestión fundamental llevó a la creación, durante la conferencia de la IFLA de 1991 en Moscú, del Grupo de Estudio de los Requerimientos Funcionales de los Registros Bibliográficos bajo la dirección del Comité Permanente de la Sección de Catalogación de la IFLA, con representación de la Sección de Clasificación e Indización (Jouguelet, 1994). El propósito del estudio es "desarrollar un modelo que sirva de base al estudio de los atributos a la luz de las necesidades de los usuarios. El estudio sirve como una racionalización de los elementos informativos usados en los registros bibliográficos, teniendo en cuenta a todos los tipos de usuarios" (Hopkins, 1994, p. 116).

Este propósito fue especificado en un conjunto de requerimientos que, siguiendo una sugerencia realizada en el Seminario de Estocolmo, propuso el desarrollo de un modelo que debía definir las entidades de interés para los usuarios de los registros bibliográficos y los tipos de relaciones que pueden existir entre estas entidades. Un modelo de este tipo puede constituir el fundamento del trabajo subsiguiente de identificar los atributos de las entidades y las relaciones requeridas para llevar a cabo las funciones del registro bibliográfico.

Tillett (1995), al definir los aspectos teóricos y prácticos del estudio, plantea una serie de cuestiones a las que éste intenta dar respuesta: "¿qué materiales deben ser representados en una base de datos bibliográficos estructurada?", "¿cómo usamos los registros bibliográficos en la combinación de entornos manuales y tecnológicos?”, “¿qué función esperamos que realicen los registros bibliográficos?" y, "a partir de un conocimiento dado de los usos del registro, ¿qué elementos esenciales o atributos deben reflejarse en el registro bibliográfico?".

Tres consultores estadounidenses (Elaine Svenonius, Barbara Tillett y Ben Tucker) han trabajado en este proyecto bajo la dirección de Olivia Madison. En Nueva Delhi (1992), la sección aprobó los términos de referencia preparados por este grupo. El grupo de estudio se amplió, especialmente con comentaristas. En Barcelona (1993), Olivia Madison renunció como jefe (aunque continuó trabajando como miembro del grupo) y fue reemplazada por Nancy John. Tucker, que había presentado también su renuncia como consultante, fue sustituido por Tom Delsey. En noviembre de 1993, los consultores y el grupo de estudio celebraron un encuentro de trabajo de dos días en Chicago (Jouguelet, 1994).

En este encuentro se revisó completamente el borrador del estudio y se asig-

Scire. 2 : 1 (en.-jun. 1996). 
naron responsabilidades para finalizar y distribuir el informe y para establecer un calendario para la revisión internacional (Tillett, 1995). Durante la Conferencia de la IFLA celebrada en La Habana en 1994 tuvo lugar una reunión de trabajo donde Tillett hizo un planteamiento general del estudio y se presentaron cuatro perspectivas internacionales sobre éste: la nórdica (Inger Cathrine Spangen), la española (Assumpció Estivill), la francesa (Maria Witt) y la norteamericana (Ingrid Parent), coincidiendo todas ellas en su apoyo al estudio (Madison, 1995, p. 2).

Estivill (1995) sintetizó sus impresiones sobre el estudio señalando que es impecable desde el punto de vista teórico. En su opinión, "aporta elementos nuevos y sistematiza otros, ofreciendo una base sólida sobre la que empezar a revisar los códigos catalográficos actuales y a rediseñar los formatos MARC existentes". Esto puede significar una mejora importante en la forma de funcionamiento del catálogo y en la sistematización de la presentación de los registros en línea. Sin embargo, la autora confiesa alguna reticencia con los condicionamientos económicos que deberán enfrentar las bibliotecas en la práctica. Al preguntarse por el significado del posible desarrollo de la teoría en la práctica desde una perspectiva económica señala que es probable que el análisis de las entidades bibliográficas en relación con la forma en que se utilizan los registros bibliográficos provocará una mayor sistematización del registro MARC y la eliminación de la información redundante. Quizás incluso se considere oportuno reducir la cantidad de datos descriptivos. Sin embargo, no parece probable esta reducción de información a partir de las indicaciones que se dan en el estudio y el trabajo llevado a cabo por la IFLA. Más aún, la eliminación de información descriptiva del registro no representa ahorros significativos.

Además, la función vinculante más poderosa en los términos establecidos en el estudio puede significar costes añadidos en la creación de los registros. Mientras esta función debe llevarse a cabo por medio de puntos de acceso y mecanismos creados para expresar relaciones y vincular registros relacionados, el establecimiento de puntos de acceso es la más costosa, la que más tiempo consume y la menos transferible de las tareas catalográficas. Aun en el caso de los puntos de acceso, al menos es posible intercambiar estos datos sin problemas en el mismo país o en el mismo idioma y bajo una práctica catalográfica común. Sin embargo, cuando expresamos relaciones y expresamos vínculos entre los registros, la transferencia automática de este tipo de información no será posible en algunos casos, incluso en las circunstancias más favorables. Si las conexiones se hacen a nivel local, deberán ser revisadas a nivel de red y viceversa.

Ya hemos señalado que el Estudio de los Requerimientos Funcionales pone las bases para replantear las reglas de catalogación y los formatos bibliográficos. Pero esto deberá hacerse de forma coordinada y serán necesarios los acuerdos

Scire. 2 : 1 (en.-jun. 1996). 
internacionales. Como señala Estivill, "la comunidad bibliotecaria mundial ha sido muy receptiva a las normas de catalogación internacionales desde hace más de treinta años. Esta tendencia continuará probablemente en el futuro porque el acuerdo normativo es la única forma de compartir los registros bibliográficos, y el incremento del uso de los sistemas automatizados y las redes internacionales facilita el intercambio y la copia de éstos" (ibidem).

\section{El proyecto de registro básico: una norma alternativa.}

En 1993, en un esfuerzo para reformar y extender el alcance de los programas cooperativos nacionales, el Cooperative Cataloging Council (CCC) estadounidense (5) nombró un grupo de trabajo para que recomendase unas normas de catalogación económicamente rentables que pudiesen ser aceptadas por un segmento de la comunidad cooperativa tan amplio como fuese posible. Entre las recomendaciones finales del grupo de trabajo estaba la propuesta de un modelo de norma bibliográfica de nivel básico. Esta norma presenta un nivel de catalogación flexible, menor que completo, que pone el acento en las necesidades locales y en las decisiones lógicas. Fue desarrollada en respuesta a lo inadecuado de las normas de nivel mínimo y de nivel completo. La primera fue considerada rentable pero de utilidad limitada para la catalogación cooperativa, mientras que la segunda es frecuentemente muy cara. La norma de nivel básico representa una tercera opción que posee alguna de las ventajas de ambos niveles de catalogación, pero sin sus inconvenientes.

Los registros básicos difieren en cuatro aspectos de los registros de nivel mínimo:

1. Los valores de los campos USMARC de longitud fija deben codificarse totalmente.

2. La norma de nivel básico prescribe una variedad más completa de elementos descriptivos.

3. Mientras ofrece menos puntos de acceso que los registros de nivel completo, los registros de nivel básico especifican fórmulas flexibles para los asientos secundarios y los encabezamientos de materia.

4. El registro básico obliga a que se dé un número clasificatorio, pero no especifica que la clasificación pueda reflejar otros sistemas que no sean reconocidos por el USMARC.

Respecto a los registros de nivel completo, la norma de registro básico difiere en dos cuestiones importantes:

1. Prescribe menos elementos de datos e intenta reducir la redundancia del registro de nivel completo. Las notas prescritas están severamente res-

Scire. 2 : 1 (en.-jun. 1996). 
tringidas por el principio de que sólo deben requerirse las notas que sean esenciales para la identificación de un documento. En el registro básico se requieren pocos asientos secundarias y no más de uno o dos encabezamientos de materia.

2. El registro básico permite una mayor flexibilidad a las agencias locales, ya que pone el acento en el sentido común para su creación. Puesto que es un registro dinámico que puede mejorarse cuando sea necesario, no es necesario que las bibliotecas contribuyentes elaboren un registro extenso que cubra todas las necesidades de los diferentes tipos de instituciones.

Actualmente se encuentra en fase de implementación y, como señala Cromwell (1994, p. 424), "el registro básico es sólo la primera etapa en un proceso sistemático de definición de un nuevo entorno catalográfico".

\section{Notas.}

(1) Crawford ha señalado, en este sentido, que "es increíblemente arrogante asumir que lo que la gente quiere es irrelevante porque nosotros sabemos lo que necesita" (Crawford, 1993, p. 254).

(2) Prins y De Gier lo han expresado de una forma concisa y directa: "Los bibliotecarios se preocupan más de sí mismos que de los propios usuarios” (Prins; De Gier, 1992, p. 109).

(3) Aunque muchos de los responsables de la elaboración de códigos tuvieron presentes los intereses de los usuarios de la biblioteca cuando escribieron sus códigos (algunos indican en la introducción de estos que la extensión de las necesidades de los usuarios afectó a sus reglas), generalmente no estudiaron la incorporación de métodos empíricos para investigar los requerimientos de los usuarios, qué necesidades eran las que hacían necesaria para ellos la consulta de los catálogos en primer lugar, y las formas en que los usuarios consultan actualmente los catálogos esperando encontrar respuestas que satisfagan sus necesida-des, esto es, su conducta de búsqueda en el catálogo. Una razón para esto es que los métodos empíricos para el estudio sistemático de las necesidades y la conducta de los usuarios eran inexistentes en el período de tiempo en que se compilaron los códigos. Pero aunque los métodos de encuesta aplicables al uso del catálogo han sido accesibles desde hace varias décadas, actualmente se han hecho estudios empíricos independientes de la utilización que hacen los usuarios de la biblioteca de catálogos de todo tipo, pero estos métodos y su aplicación no han influenciado significativamente la estructura o contenido de algún código (Lancaster, 1977, p. 63).

(4) Existe una recopilación bibliográfica, un poco antigua ya, sobre esta cuestión (Seal, 1978).

(5) El Cooperative Cataloging Council (CCC) fue creado en un encuentro de la ALA en enero de 1993 por representantes de la Library of Congress, OCLC, RLG y representantes de bibliotecas públicas y universitarias participantes en programas de coopera-

Scire. 2 : 1 (en.-jun. 1996). 
ción cooperativa. Sus objetivos son: (1) desarrollar un plan estratégico para implementar la misión y los objetivos ya identificados, (2) identificar aspectos y cuestiones que necesitan ser tratadas, (3) determinar el mejor forum para tratar estas cuestiones, (4) establecer pequeños grupos de trabajo monográficos cuando sea necesario, (5) determinar los mecanismos para solicitar y difundir los comentarios, (6) establecer un calendario para el proceso de planificación, y (5) hacer recomendaciones para la implementación a la Library of Congress como coordinador del esfuerzo (Anderson, 1993, p. 65).

\section{Referencias.}

Abrera, J. B. (1982). Bibliographic structure possibility set: a quantitative approach for identifying users' bibliographic information needs. // Library Resources \& Technical Services. 26:1 (1982) 47-51.

Anderson, D. (Dorothy) (1989). Standard practices in the preparation of bibliographic records. Rev. ed. London: IFLA UBCIM Programme, 1989.

Anderson, G. (1993). Symmetry and extrapolation: passion and precision : Cooperative cataloging at the beginning of the 21 st century. // Cataloging \& Classification Quarterly. 17:3/4 (1993) 39-73.

Beheshti, J. (1992). Browsing through public access catalogs. // Information Technology and Libraries. 11:2 (1992) 220-228.

Boll, J. J. (1990). The future of AACR2. // Cataloging \& Classification Quarterly. 12:1 (1990) 3-34.

Bourdon, F. (1993). International cooperation in the field of authority data: an analytical study with recommendations. Translated from the French by Ruth Webb. München [etc.]: Saur, 1993. (UBCIM Publications. New Series, 11).

Bruce, H. W. (1994). A cognitive view of the situational dynamism of user-centered relevance estimation. // Journal of the American Society for Information Science. 45:3 (1994) 142-148.

Brunt, R. M. (1992). The code and the catalogue: a return to compatibility. // Library Review. 41:3 (1992) 21-32.

Bryant, P. (1988). End user requeriments of bibliographic records. // Greenwood, Derek (comp.). Bibliographic records in the book world: needs and capabilities: proceedings of a seminar held on 27-28 November 1987, at Newbury. London: British National Bibliography, 1988. p. 13-19.

Bryant, P. (1995). Quality of a national bibliographic service: in the steps of John Whytefeld : an admirable cataloguer. // International Cataloguing and Bibliographic Control. 24:2 (1995) 29-32.

Buckland, M. K. (1988). Bibliography, library records, and the redefinition of the Library of Congress. // Library Resources \& Technical Services. 32:4 (1988) 299-311.

Buckland, M. (1992). Redesigning library services: a manifesto. Chicago; London: American Library Association, 1992.

Busquets, D. (1992). Los niveles alternativos en los estándares bibliográficos. // Revista española de documentación científica. 15:2 (1992) 154-173.

Scire. 2 : 1 (en.-jun. 1996). 
Butcher, J. E. (1988). Suppliers' capabilities: BL Bibliographic Services. // Greenwood, Derek (comp.). Bibliographic records in the book world: needs and capabilities: proceedings of a seminar held on 27-28 November 1987, at Newbury. London: British National Bibliography, 1988. p. 103-108.

Byrum, J. D. (1992). Panel discussion on second day's papers. // Seminar on Bibliographic Records (1990. Stockholm). Seminar on Bibliographic Records: proceedings of the seminar held in Stockholm, 15-16 August 1990, and sponsored by the IFLA UBCIM Programme and the IFLA Division of Bibliographic Control. Edited by Ross Bourne. München [etc.]: Saur, 1992. p. 136-140.

Carrión Gútiez, M. (1987). Manual de bibliotecas. Salamanca; Madrid: Fundación Germán Sánchez Ruipérez, 1987.

Chevallier, A. (1992). Information, bibliographic records and library needs. // Seminar on Bibliographic Records (1990. Stockholm). Seminar on Bibliographic Records: proceedings of the seminar held in Stockholm, 15-16 August 1990, and sponsored by the IFLA UBCIM Programme and the IFLA Division of Bibliographic Control. Edited by Ross Bourne. München [etc.]: Saur, 1992. p. 33-47.

Clement, H. (1992). Opening remarks by chairman. // Seminar on Bibliographic Records (1990. Stockholm), Seminar on Bibliographic Records: proceedings of the seminar held in Stockholm, 15-16 August 1990, and sponsored by the IFLA UBCIM Programme and the IFLA Division of Bibliographic Control. Edited by Ross Bourne. München [etc.]: Saur, 1992. p. 1-2.

Crawford, W. (1993). The future online catalog: a single view of multiple databases. // Information Technology and Libraries. 12:2 (1993) 253-254.

Cromwell, W. (1994). The core record: a new bibliographic standard. // Library Resources \& Technical Services. 38:4 (1994) 415-424.

Currency and coverage [Consultive paper] (1987). London: British Library Bibliographic Services, 1987.

Dempsey, L. (1989). Bibliographic records: use of data elements in the book world. Bath: Bath University Library, 1989.

Duke, J. K. (1989). Access and automation: the catalog record in the age of automation. // Svenonius, Elaine (ed.). The conceptual foundations of descriptive cataloging. San Diego [etc.]: Academic Press, 1989. p. 117-133.

Esman, M. (1991). Guest editorial. // Technical Services Quarterly. 9:2 (1991) 3-5.

Estivill, A. (1995). The Functional Requirements of Bibliographic Records Study: comments on the study. // International Cataloguing and Bibliographic Control. 24:3 (1995) 44-45.

Fox, M. A.; Preece, B. G. (1991). Upgrading minimal level monographic records: a study and conclusions. // Technical Services Quarterly. 8:4 (1991) 25-35.

García Caro, C. (1994). Los catálogos en línea de acceso público (OPAC). // Pinto Molina, María (ed.). La catalogación de documentos: teoría y práctica. Madrid: Síntesis, 1994. p. 419-431.

Gorman, M. (1968). A study of the rules for entry and heading in the AngloAmerican cataloging rules, 1967. London: Library Association, 1968.

Scire. 2 : 1 (en.-jun. 1996). 
Graham, W. G. (1988). The future of scientific publishing. London: British Library, 1988.

Gredley, E.; Hopkinson, A. (1990). Exchanging bibliographic data: MARC and other international formats. Ottawa: Canadian Library Association; London: The Library Association; Chicago: American Library Association, 1990.

Hagler, R. (1991). The bibliographic record and information technology. 2nd ed. Twickenham: Adamantine Press, 1991.

Hancock-Beaulieu, M. (1991). Bibliographic standards and the online catalogue user. // McIlwaine, I. C. (ed.), Standards for the international exchange of bibliographic information: papers presented at a course held at the School of Library, Archive and Information Studies, University College London, 3-18 August 1990. London: The Library Association, 1991. p. 87-91.

Handman, G. (1991). I lost it (or found it) at the movies: public service implications of minimal-level cataloging of audiovisual materials. // Technical Services Quarterly. 9:2 (1991) 39-47.

Harris, G. (1989). Historic cataloging costs, issues, and trends. // Library Quarterly. 59:1 (1989) 1-21.

Hopkins, J. (1994). American Library Association Midwinter Meeting, Los Angeles, California, February 1994: the ALCTS/CCS Committee on Cataloging: Description and Access (CC:AA) Met Saturday, February 5, 2:00-5:30 p.m. and Monday, February 7, 9:30 a.m.-1:10 p.m. // Cataloging \& Classification Quarterly. 19:2 (1994) 114-125.

Horny, K. L. (1991). What you see is what you get (so what do you get?): an administrator looks at minimal-level cataloging. // Technical Services Quarterly. 9:2 (1991) 719.

Hufford, J. R. (1991). The pragmatic basis of catalog codes: has the user been ignored?. // Cataloging \& Classification Quarterly. 14:1 (1991) 27-38.

Hyslop, C. F. (1994). PromtCat prototype: accelerating progress in technical services. // The future is now: the changing face of technical services: proceedings of the OCLC Symposium ALA Midwinter Conference, February 4, 1994. Dublin, OH: Online Computer Library Center, 1994. p. 33-38.

Jouguelet, S. (1994). Section on Cataloguing: review of the work 1993/1994. // IFLA 60th General Conference, 21-27 August 1994, Havana, Cuba. (040-BIBCO-4-E).

Karamac, B. (1992). Panel discussion on first day's papers. // Seminar on Bibliographic Records (1990. Stockholm). Seminar on Bibliographic Records: proceedings of the seminar held in Stockholm, 15-16 August 1990, and sponsored by the IFLA UBCIM Programme and the IFLA Division of Bibliographic Control. Edited by Ross Bourne. München [etc.]: Saur, 1992. p. 68-76.

Knutson, G. (1993). The year's work in descriptive cataloging, 1992. // Library Resources \& Technical Services. 37:3 (1993) 261-275.

Lambrecht, J. H. (1992). Minimal level cataloguing by national bibliographic agencies. München [etc.]: K. G. Saur, 1992.

Lancaster, F. W. (1977). Studies of catalog use. // The measurements and evaluation of library services. Arlington, VA: Information Resources Press, 1977. p. 19-72.

Scire. 2 : 1 (en.-jun. 1996). 
Leazer, G. H. (1992). An examination of data elements for bibliographic description: toward a conceptual schema for the USMARC format. // Library Resources \& Technical Services. 36:2 (1992) 189-208.

Lehmann, K.-D. (1992). Links in the information chain. // Seminar on Bibliographic Records (1990. Stockholm). Seminar on Bibliographic Records: proceedings of the seminar held in Stockholm, 15-16 August 1990, and sponsored by the IFLA UBCIM Programme and the IFLA Division of Bibliographic Control. Edited by Ross Bourne. München [etc.]: Saur, 1992. p. 81-100.

Line, M. B. (1988). Sastifying bibliographic needs in the future :from publisher to user. Catalogue \& Index: 90/91 (1988) 10-14.

Lipow, A. G. (1979). The catalog in a research library and alternatives to it. // Freedman, Maurice J.; Malinconio, S. Michael (eds.). The nature and future of the catalog: proceedings of the ALA Information Science and Automation Division's 1975 and 1977 Institutes on the Catalog. Phoenix: Oryx Press, 1979. p. 240-256.

Madison, O. M. A. (1995). 60th IFLA Conference report, Havana, Cuba. // SCATNews. 5 (1995) 2-3.

Magán Walls, J. A. (1995). La descripción bibliográfica hoy: sugerencias para el aprovechamiento de las posibilidades de las nuevas tecnologías. // Magán Walls, José Antonio (coord.). Tratado básico de biblioteconomía. Madrid: Editorial Complutense, 1995. p. 185-199.

Neville, H. H. (1981). Computers and the language of bibliographic descriptions. // Information Processing \& Management. 17:3 (1981) 137-148.

O'Brien, A. (1990). Relevance as an aid to evaluation in OPACs. // Journal of Information Science. 16 (1990) 265-271.

Osiobe, S. A. (1987). Use and relevance of information on the card catalogue to undergraduate students. // Library Review. 36:4 (1987) 261-267.

Park, T. K. (1993). The nature of relevance in information retrieval: an empirical study. // Library Quarterly. 63:3 (1993) 318-351.

Patton, G. (1991). OCLC's long association with less than full cataloging. // Technical Services Quarterly. 9:2 (1991) 21-30.

Piggott, M. (1988). A topography of cataloguing: showing the most important landmarks, communications and perilous places. London: Library Association, 1988.

Piggott, M. (1990). The cataloguer's way through AACR2: from document receipt to document retrieval. London: Library Association, 1990.

Prins, H.; De Gier, W. (1992). Image, status, and reputation of librarianship and information work. IFLA Journal. 18:2 (1992) 108-118.

Question and answers (1994). // The future is now: the changing face of technical services: proceedings of the OCLC Symposium ALA Midwinter Conference, February 4, 1994. Dublin, OH: Online Computer Library Center, 1994. p. 43-45.

Reglas de catalogación. Ed. refundida y rev. Madrid: Dirección General del Libro, Archivos y Bibliotecas, 1995.

Seal, A. (1978). Bibliography on the content of catalogue entries. Bath: Bath University, Programme of Catalogue Research, 1978.

Scire. 2 : 1 (en.-jun. 1996). 
Seal, A. (1983). Experiments with full and short entry catalogues: a study of library needs. // Library Resources \& Technical Services. 27:2 (1983) 144-155.

Seal, A.; Bryant, P.; Hall, C. (1982). Full and short entry catalogues: library needs and uses. Bath: Centre for Catalogue Research, Bath University Library, 1982.

Soules, A.; Lucas, J.; Pritts, S. (1992). Compromises in the management of working papers. // Library Resources \& Technical Services. 36:4 (1992) 478-486.

Stubley, P. (1988). User requirements in reader services. // Greenwood, Derek (comp.). Bibliographic records in the book world: needs and capabilities: proceedings of a seminar held on 27-28 November 1987, at Newbury. London: British National Bibliography, 1988. p. 46-52.

Swanson, D. R. (1986). Subjective versus objective relevance in bibliographic retrieval systems. // Library Quarterly. 56:4 (1986) 389-398.

Tillett, B. B. (1989). Bibliographic structures: the evolution of catalog entries, references, and tracings. // Svenonius, Elaine (ed.). The conceptual foundations of descriptive cataloging. San Diego [etc.]: Academic Press, 1989. p. 149-165.

Tillett, B. B. (1995). IFLA Study on the Functional Requirements of Bibliographic Records: theoretical and practical foundations. // International Cataloguing and Bibliographic Control. 24:3 (1995) 43-44.

Trickey, K. V. (1995). A universe in search of a structure: the cataloguer in the network, CIG Under One UmbrelLA Three, UMIST, 23-25 June, 1995: the view from the chair. // Catalogue \& Index. 116 (1995) 5-6.

Tyckoson, D. (1991). Access vs. ownership: changing roles for librarians. // The Reference Librarian. 34 (1991) 37-45.

Van Houten, S. (1981). In the iron age of cataloging. // Library Resources \& Technical Services. 25:4 (1981) 362-373.

Yao, Y. Y. (1995). Measuring retrieval effectiveness based on user preference of documents. // Journal of the American Society for Information Science. 46:2 (1995) 133145.

Yatsko, V. A. (1991). Semantic structure of a bibliographic description. // Automatic Documentation and Mathematical Linguistics. 25:3 (1991) 46-49.

Scire. 2 : 1 (en.-jun. 1996). 